Legajos, núm. 10, 2016, pp. 45-67.

\title{
El Tribunal para Menores Infractores en las primeras décadas del siglo $X X$.
}

\section{ZOILA SANTIAGO ANTONIO.}

Cita:

ZOILA SANTIAGO ANTONIO (2016). EI Tribunal para Menores Infractores en las primeras décadas del siglo XX. Legajos, (10), 45-67.

Dirección estable: https://www.aacademica.org/zoila.santiago/5

ARK: https://n2t.net/ark:/13683/pr42/mw8

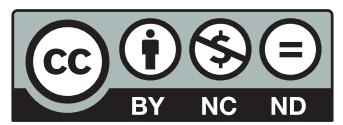

Esta obra está bajo una licencia de Creative Commons.

Para ver una copia de esta licencia, visite

https://creativecommons.org/licenses/by-nc-nd/4.0/deed.es.

Acta Académica es un proyecto académico sin fines de lucro enmarcado en la iniciativa de acceso abierto. Acta Académica fue creado para facilitar a investigadores de todo el mundo el compartir su producción académica. Para crear un perfil gratuitamente o acceder a otros trabajos visite: https://www.aacademica.org. 


\section{El Tribunal para Menores Infractores en las primeras décadas del siglo $\mathrm{XX}$}

The Juvenile Offenders Court in the Early Twentieth Century

\section{Zoila Santiago Antonio}

Colegio de Historia de Facultad de Filosofía y Letras

Benemérita Universidad Autónoma de Puebla zoilasan09@gmail.com

\section{Resumen}

Este texto examina algunos de los problemas que enfrentó el Tribunal para Menores Infractores en las primeras décadas del siglo Xx. Dicha institución fue creada para lograr corregir el comportamiento de los niños y jóvenes que iniciaban su camino por la delincuencia, pero el alto nivel de reincidencia pone en duda su buen funcionamiento. A pesar de no lograr completamente su objetivo, el Tribunal pudo resolver algunos problemas de forma inmediata, como fue el abandono infantil. La sobrepoblación, la falta de personal capacitado, las instalaciones deterioradas o el maltrato fueron algunas de las situaciones por las que tuvieron que atravesar algunos niños y jóvenes que estuvieron en esta institución.

Palabras clave: delincuencia, Tribunal para Menores Infractores, infancia

\section{Abstract}

This article examines some of the problems the Court for Juvenile Offenders faced during the early decades of the twentieth century. The institution was created to correct the behavior of children and youth who were beginning to turn to crime - a goal belied by high levels of recidivism. Although it did not fully achieve its objective, the court was able to solve immediately some problems such as child abandonment. Still, children and young people in this institution often endured overcrowding, lack of trained personnel, deteriorated installations, and abuse.

Keywords: delinquency, Juvenile Court, childhood 


\section{Introducción}

A principios del siglo $\mathrm{xx}$, la infancia comenzó a ser un tema discutido en diferentes países, tanto de Europa como de América Latina. Se comenzaron a realizar congresos con el objetivo de entender el desarrollo integral de los niños. A partir de estos eventos se elaboraron una serie de políticas proteccionistas hacia la infancia. Beatriz Alcubierre menciona, por ejemplo, que los temas abordados dentro de los congresos panamericanos muestran que las clases acomodadas de los países que participaron compartían un mismo ideal de niñez, que trataron de implantar al resto de la sociedad. Pero sus esfuerzos se vieron limitados y tuvieron que enfrentarse a la dura realidad: su ideal de infancia se contrapuso con la de menores pertenecientes a las clases populares, es decir, niños abandonados, trabajadores y sin educación, ${ }^{1}$ que vivían en países con un alto nivel de mortalidad infantil, como era el caso de México.

Por otro lado, Patience Shell señala que, tanto discursiva como simbólicamente, el niño se convirtió en un medio para representar el potencial nacional, ya que personificaba, en el discurso oficial, el futuro de la nación. ${ }^{2}$ Este interés alcanzó, incluso, a los menores infractores que se encontraban fuera del ideal de infancia de las clases posrevolucionarias, pero a los que se trató de integrar al programa de reconstrucción nacional iniciado por los gobiernos posrevolucionarios, por medio de una serie de eventos e instituciones que aparecieron con el fin de crear al "nuevo hombre", la "nueva mujer" y el "nuevo niño". De tal forma, el niño fue convertido en actor nacional, y por consiguiente, su preparación como ciudadano era de suma importancia para el Estado, de ahí que las autoridades se centraran en el modelo familiar. La intervención del Estado dentro del ámbito privado, en este caso de la familia, tuvo el fin de proteger el desarrollo integral de las futuras generaciones. Por tal motivo, era importante regular la vida de los niños por la vía de los centros educativos o penitenciarios,

\footnotetext{
'Alcubierre Moya, "La infancia", p. 123.

${ }^{2}$ Shell, "Nationalizing Children", pp. 563, 587.
} 
como fue el Tribunal para Menores Infractores del Distrito Federal. ${ }^{3}$ Las políticas proteccionistas hacia la infancia tuvieron como objetivo intervenir en la vida de los individuos desde el nacimiento hasta la juventud por medio de la educación y de la asistencia social, para obtener un determinado "tipo de ciudadano". 4

Estas políticas se insertaron dentro del programa de reconstrucción nacional erigido por los gobiernos posrevolucionarios de las primeras décadas del siglo xx. El cual incluía la reconstrucción material, social y cultural de la población, en donde se pretendía cambiar costumbres y hábitos de sus habitantes que se consideraban un obstáculo para el progreso y la modernidad del país. Urías Horcasitas señala que los gobiernos posrevolucionarios "pusieron en marcha un programa de ingeniería social cuyo objetivo fue 'mejorar' la calidad de la población para hacer surgir una nueva sociedad fisica y moralmente 'regenerada". ${ }^{5}$ De acuerdo con Beatriz Urías, este proyecto tuvo dos objetivos, por un lado pretendió la integración de los "grupos étnicos al resto de la población a través del mestizaje, la españolización y la educación"; y por el otro, "establecer medidas profilácticas en el ámbito de la vida reproductiva a fin de controlar la herencia degenerativa que provocaba el nacimiento de individuos con inclinación hacia el alcoholismo, la drogadicción, las enfermedades mentales, las desviaciones sexuales y las tendencias criminógenas". ${ }^{6}$

En este contexto, para algunos reformadores sociales la delincuencia infantil representaba un peligro para la sociedad, porque obstaculizaba este proyecto de reconstrucción nacional. Por ejemplo, Ramón Beteta, ${ }^{7}$ en su estudio sobre La mendicidad en México, publicado en 1931, señaló

${ }^{3}$ Shell, "Nationalizing Children", p. 565.

${ }^{4}$ Netto Nunes, "La infancia latinoamericana", p. 275.

${ }^{5}$ Urías Horcasitas, "Degeneración”, p. 38.

${ }^{6}$ Urías Horcasitas, "Degeneración”, p. 39.

${ }^{7}$ Ramón Beteta, durante su trayectoria profesional, ocupó varios puestos públicos, estuvo en la Secretaría de Salubridad y Asistencia, y en la Secretaría de Educación. Además, fue consejero de los Ferrocarriles y secretario de Hacienda. También fungió como embajador de México en las embajadas de Italia y Grecia. Beteta, Farano, pp. 7-8. 
que el "crimen representa, a primera vista, el índice más significativo en la desorganización social y de falta de control en una comunidad". ${ }^{8}$ De tal forma, el interés principal de la delincuencia no sólo se centraba en lo perjudicial que era para la organización social de un país, en este caso, sino de los miles de niños que se desviaban del "buen camino", lo que significaba la pérdida de los futuros ciudadanos que México necesitaba para lograr sus metas. A esta preocupación se sumaron otras como el desamparo en que se encontraban los menores delincuentes, la falta de instituciones y reglamentos que se encargaran de juzgarlos según su edad.

Los discursos que aparecieron para explicar la delincuencia infantil, criminalizaron la pobreza y ciertas conductas de la mayoría de la población de la ciudad de México. Susana Sosenski señala que "los individuos fueron distinguidos no sólo por sus actos delictivos sino por sus 'predisposiciones' a estos actos. Así se crearon sospechosos y se criminalizaron varias prácticas de los sectores populares". ${ }^{9}$

La mayoría de los intelectuales hicieron énfasis en los grupos sociales que consideraban como peligrosos, entre los que se encontraban los niños abandonados o que tenían familias consideradas como "desorganizadas". Para ellos se implementaron toda una serie de iniciativas de carácter público y privado que tenían "una visión asistencial sustentada en mirarlos como 'objetos de protección' y 'necesitados de tutela", pero a su vez eran vistos como "víctimas, inadaptados peligrosos y depositarios de todos los males". ${ }^{10}$ Es decir, que mientras en el discurso se abogaba por proteger a este sector en específico, en el fondo se les temía, por ello se deseaba cambiar y moldear su comportamiento con ayuda de las diversas instituciones que se reformaron o aparecieron, en las primeras décadas del siglo xx, con el fin de proteger a los más desvalidos.

Asimismo, la situación económica y social que rodeaba a los menores permitió la intervención del Estado dentro de sus familias a partir de

\footnotetext{
${ }^{8}$ Beteta, La mendicidad, p. 74.

9 Sosenski, "Infancia", p. 15. Para profundizar más sobre el tema consultar a Piccato, Ciudad.

${ }^{10}$ Pérez García, "La infancia callejera", p. 298.
} 
diversas instituciones, como fue el Tribunal para Menores Infractores (1926). Sus problemas fueron minimizados y se creyó que entre los factores causantes de la delincuencia infantil estaban la desorganización familiar, la herencia, la falta de educación, entre otros. Pero, la situación económica y social de las familias y de los menores fueron consecuencias de la migración, de la falta de viviendas, de la carencia de los servicios públicos, del proceso de industrialización, de la pobreza. Estos elementos impidieron que las familias de los menores fueran consideradas como ideales para su protección y crianza como futuros ciudadanos.

El objetivo de este texto es comprender si el Tribunal para Menores logró la regeneración e incorporación de estos niños y jóvenes a la sociedad, además, señalar de forma general la situación del Tribunal y de algunos de sus establecimientos correccionales entre 1927 y 1937. Con la aparición de esta institución se creyó que se estaba más cerca de la tan anhelada civilización y progreso "occidental", pero el tiempo se encargó de mostrar qué tan lejos estuvieron de lograr sus objetivos.

La información que se presentará a continuación fue el resultado del análisis de 2162 expedientes consultados del Consejo Tutelar para Menores del Distrito Federal, que se encuentra en el Archivo General de la Nación. El objetivo de la investigación era consultar todos los expedientes desde febrero de 1927 hasta diciembre de 1937, pero debido al tiempo limitado sólo se pudieron consultar de la caja uno a la 10 que corresponden de febrero de 1927 a diciembre de 1929, y de la caja 20 a la caja 51 que corresponden al lapso de diciembre de 1932 hasta junio de 1937. La decisión de dejar 10 cajas sin consultar fue un tanto al azar, se trató de calcular que éstas coincidieran entre 1932 y 1933, es decir, que estuvieran en medio del periodo de estudio, lo cual no sucedió. Pero, la información de las cajas no consultadas puede variar poco la información presentada aquí, debido a que estimamos que las cajas correspondientes a los diez años que abarca la investigación, son alrededor de cincuenta y seis, y nosotros consultamos 41, lo que correspondería a $74.54 \%$ de nuestro universo total, mucho más de $10 \%$ o $15 \%$ que se pide para la muestra de un universo específico. 


\section{El Tribunal para Menores Infractores}

En 1920 se realizó en la ciudad de México el Primer Congreso del Niño, en el que participaron una infinidad de personas de diferentes especialidades con el objetivo de mejorar las condiciones de la niñez mexicana. En este primer Congreso fueron diversos los temas que se abordaron, los cuales estuvieron organizados en las siguientes secciones: Eugenesia, Pediatría Médica, Pediatría Quirúrgica, Higiene, Enseñanza y Legislación Infantil. Todas ellas estaban destinadas a elaborar un programa que lograra el mejoramiento de la niñez mexicana, así que en cada una se mencionaban los problemas o las deficiencias que aquejaban a los niños y a las madres, y cómo se podían mejorar.

Este evento fue sumamente importante, primero porque se ponía en el centro de interés de hombres y mujeres, al niño mexicano. Segundo, porque a partir de los problemas denunciados se propusieron soluciones, además de hacer un llamado a la sociedad para la participación de todos. Algunas de las propuestas que se llegaron a concretar, a partir de las ideas expuestas por los congresistas fueron: los cursos impartidos de puericultura para mujeres jóvenes, la construcción de parques y la formación de brigadas de sanidad para visitar a las mujeres embarazadas en los barrios de la ciudad de México, la construcción del primer Centro de Higiene Infantil en 1922, y la creación del Tribunal para Menores en la ciudad de México en $1926 .{ }^{11}$

Así que la creación del Tribunal para Menores respondió a varias preocupaciones de los reformadores sociales de la época. Por un lado, resolver el problema del abandono infantil causado por el aumento de la migración, o por la desintegración de las familias debido a la muerte o el abandono de uno o ambos padres. Por otro lado, solucionar la falta de instancias legales encargadas de juzgar a los niños y jóvenes delincuentes, para evitar que tanto niños como adultos convivieran en un mismo espacio, y que no fueran tratados de la misma forma.

En las casi dos primeras décadas del siglo xx, el código penal que seguía vigente en el país era el de 1871. De acuerdo con este código,

${ }^{11}$ Stern, "Madres conscientes", pp. 300-301. 
se estableció la responsabilidad de los menores según su edad y tomando en cuenta la falta o no de discernimiento. De esta manera, el artículo 34 excluyó de responsabilidad penal a los niños menores de nueve años; también fueron descartados los mayores de nueve años y menores de catorce, en caso de que el acusador no pudiera probar que el menor había actuado con discernimiento, es decir, que era consciente o no de sus actos. Pero, aquél que era mayor a catorce años se consideraba que había actuado con discernimiento y las penas impuestas eran entre la tercera parte y la mitad de la condena que se le aplicaba a un adulto. ${ }^{12}$

Por tal razón, los reformadores sociales pidieron la creación de un Tribunal para Menores con el fin de "juzgar a los menores y establecer la forma en que éstos, más que extinguir una condena vayan a reformarse en sus hábitos y costumbres" para poder ser integrados a la sociedad. ${ }^{13}$ Además, denunciaron el mal estado en que se encontraban los establecimientos correccionales existentes en ese momento, se decía que eran "verdaderas cárceles en donde los niños delincuentes, después de sufrir castigos corporales y torturas morales, contraen hábitos perniciosos" y "transforman al niño, delincuente ocasional e irresponsable casi siempre, en delincuente responsable y habitual", debido a su convivencia con otros infractores. ${ }^{14}$

El 8 de octubre de 1926 apareció el "Reglamento para la calificación de los infractores menores de edad en el Distrito Federal" en el Diario Oficial. Éste sólo facultaba al Tribunal para actuar cuando los menores de 16 años cometieran algún delito sancionado en el código penal, para estudiar las solicitudes de los menores de edad sentenciados por los tribunales del orden común que deseaban obtener reducción o conmutación de su pena; conocer los casos de vagancia y mendicidad de menores de dieciocho años; intervenir, a solicitud de los padres o tutores, en los casos de menores de dieciséis años denominados como "incorregibles". ${ }^{15}$

\footnotetext{
${ }^{12}$ Ceniceros y Garrido, La delincuencia, pp. 18, 247.

${ }^{13}$ Memorias del Primer, p. 366.

${ }^{14}$ Memorias del Primer, p. 369.

15 "Reglamento para la calificación de los infractores menores de edad en el Distrito Federal", Diario Oficial, Ciudad de México, 8 octubre de 1926, pp. 14-16.
} 
La inauguración del establecimiento fue el 7 de diciembre de 1926, pero entró en funcionamiento hasta enero de 1927. Al evento asistieron los maestros y los jueces que integrarían el Tribunal; el gobernador del Distrito Federal, el general Serrano, además del licenciado Ortiz Tirado, quien se decía había ido a Europa para realizar estudios y observaciones sobre los tribunales de niños, y veía, al igual que los demás, "el momento de la cristalización de uno de sus ideales": ${ }^{16}$

...los niños infortunados encontrarán el amparo, muchas veces más elocuentes que el paterno, en un grupo de jueces benévolos que analizarán la vida del niño; escudriñarán sus antecedentes, observarán sus inclinaciones y lo pondrán en camino seguro - un camino de ternura y de optimismo- hacia una vida mejor; hacia una vida útil a la sociedad y a ellos, principalmente. ${ }^{17}$

Por tal motivo, esta institución tuvo como objetivo analizar las causas que llevaban a los menores a delinquir y ofrecer tratamientos psicopedagógicos para lograr su reinserción a la sociedad como un ciudadano honrado y trabajador. Así que cuando un niño o joven era presentado al Tribunal se le realizaban cuatro exámenes: el social, el pedagógico, el médico, y el psicológico para determinar el porqué de su comportamiento delictivo. Después de haber realizado los cuatro exámenes, ${ }^{18}$ en caso de que los jueces consideraran que el menor necesitaba de la protección del Estado, debido a su situación social, médica, pedagógica o psiquiátrica, y que la familia estaba incapacitada para ayudarlo, se determinaba enviarlo a alguna de sus dependencias. En un principio,

${ }^{16}$ Sin autor, "Quedó instalado el primer tribunal paternal que juzgará a los niños que cometan faltas o delitos", El Universal, segunda sección, Ciudad de México, 11 de diciembre de 1926, pp. 1 y 8.

${ }^{17}$ Sin autor, "Quedó instalado el primer tribunal paternal que juzgará a los niños que cometan faltas o delitos", El Universal, segunda sección, Ciudad de México, 11 de diciembre de 1926, pp. 1 y 8.

${ }^{18}$ Hay que aclarar que de acuerdo con los expedientes analizados, son pocos los casos en que a los menores se les aplicaban los cuatro exámenes. 
entre los establecimientos con que contó el Tribunal estuvieron la Casa Hogar, las escuelas correccionales de varones y mujeres -que eran conocidas como escuelas de orientación o "reformatorios"-, la Escuela Industrial y la Escuela Francisco I. Madero.

\section{"La Casa de Orientación para Varones no es un lugar de educación, de orientación..."}

De acuerdo a los datos proporcionados por el Departamento de Prevención Social, las infracciones que los menores cometieron entre 1927 y 1931 fueron clasificadas en seis grupos (véase la Tabla 1). Conforme a esta información, los principales delitos realizados por los menores infractores fueron en contra de la propiedad, categoría que incluía casos como: robo, estafa, daño a propiedad ajena, abuso de confianza, entre otros. Por otro lado, a partir de los 2162 datos recopilados entre 1927 y 1937, ${ }^{19}$ podemos determinar que $83.53 \%$ de los menores presentados eran varones y sólo $16.32 \%$ eran mujeres.

Asimismo, como ya habíamos mencionado, de estos 2162 expedientes, $49.12 \%$ era reincidente, y el restante $50.87 \%$ había sido presentado por primera vez al Tribunal. En cuanto a este punto, llama la atención el número tan elevado de menores que fueron presentados tantas veces al Tribunal, algunos de ellos habían salido de las escuelas o correccionales a las que habían sido enviados con anterioridad debido a su "buen comportamiento", otros, a petición de sus padres, o debido a que habían encontrado un trabajo acorde con sus "habilidades". Pero la mayoría de ellos se había fugado, ya sea del propio Tribunal, de la Casa de Observación o de las correccionales en donde se encontraban.

19 Este trabajo forma parte de mi tesis doctoral, por tal motivo, el periodo y selección de expedientes corresponden a la temporalidad de mi proyecto. Estos expedientes se pueden consultar en el Fondo del Consejo Tutelar para Menores Infractores (CTMI) que se encuentra en el Archivo General de la Nación (en adelante AGN). 


\begin{tabular}{|c|}
\hline $\begin{array}{l}\text { Causas determinantes de ingreso de entre } 4758 \text { casos } \\
\qquad(1927-1931)\end{array}$ \\
\hline $\begin{array}{l}\text { Contra la propiedad (robo, estafa, daño a propiedad ajena, abuso de confianza, com- } \\
\text { prar objetos robados, circular moneda falsa) }\end{array}$ \\
\hline $\begin{array}{l}\text { Del ambiente (estar en sitios prohibidos, jugar juegos prohibidos, escándalo en la vía } \\
\text { pública, indisciplina en el hogar, abandono del hogar, vagancia, mendicidad, prófugos) }\end{array}$ \\
\hline Contra las personas (homicidio, asalto, insultos, riña, lesiones, lesionados, secuestro) \\
\hline Protección por diversas causas (extraviados, vender drogas, intoxicación alcohólica aguda) \\
\hline Diversas causas no comprobadas \\
\hline Orden sexual (seducción, estupro y rapto, violación, faltas a la moral, prostitución) \\
\hline
\end{tabular}

Tabla 1. Datos obtenidos en Secretaría de Gobernación. Departamento de Prevención Social, Los Tribunales para Menores en el Distrito Federal y sus instituciones auxiliares, 1936.

Pero las casas de beneficencia y de corrección, como señala Alberto Trujillo Bretón, fueron establecimientos creados para tratar de proteger a los menores de edad "de cualquier tipo de contaminación que los pudiera alejar de los modelos establecidos por una moral social y familiar de carácter burgués, que veía en estos niños el porvenir de la propia familia y de la sociedad en general". Pero lejos de lograr su inserción a la sociedad como buenos ciudadanos, "estuvieron excluidos de toda oportunidad social, menos de la prisión que debía servirles a través de sus técnicas de disciplina, para intentar docilizarlos". ${ }^{20}$

De tal manera, la existencia del Tribunal para Menores no garantizaba el mejoramiento "físico o mental" de estos niños. Las causas del posible fracaso de dichos establecimientos pueden tener una infinidad de respuestas. Pero las que nosotros encontramos en los expedientes y en las denuncias que se hicieron en los años treinta, nos hablan de un mal funcionamiento y descuido de estas instituciones. Por ejemplo, en 1938 se publicó el trabajo de la reportera Carmen Madrigal sobre la situación de los menores infractores y de las instalaciones dependientes

${ }^{20}$ Trujillo Bretón, Los hijos, pp. 383-386. 
del Tribunal. Estos reportajes fueron publicados en la revista Hoy y después fueron recopilados en la obra Los menores delincuentes. Estudios sobre la situación de los Tribunales para Menores. Doctrina y realidad, con una introducción del abogado José Ángel Ceniceros. ${ }^{21}$

Carmen Madrigal denunció el tipo de vida que llevaban los menores desde la Casa de Observación hasta las correccionales de varones y mujeres. Hizo una descripción de estos lugares con lujo de detalle. Describió el abandono en que se encontraban no sólo las instalaciones del Tribunal y las correccionales sino también, el tipo de mobiliario que había, la ropa de los menores, las actividades que realizaban, pero sobre todo, describió el abandono social en que se encontraban.

Dentro de estas instalaciones - Tribunal y correccionales- no había profesores que se ocuparan de la educación de los niños; además, los talleres pocas veces funcionaban. La autora expresó que "sólo arrasando la casa y alzándola otra vez aquello tendría remedio”, refiriéndose al edificio donde se encontraba la Escuela Correccional para Varones también conocida como la "casa amarilla". De acuerdo con la descripción de Carmen Madrigal, la fachada de la institución dejaba mucho que desear al igual que el resto del edificio. En los dormitorios se podía mirar a los menores "amontonados en las angostas camas, sin una manta para cubrirse, sobre colchones destripados y en una lamentable confusión de miembros, en la que no se sabe a quién pertenece este brazo y a quién pertenece aquella pierna". ${ }^{22}$

Es interesante señalar que las observaciones que hizo Carmen Madrigal eran las mismas que los reformadores sociales habían manifestado en el Primer Congreso del Niño. De acuerdo con su denuncia, pareciera que la situación no cambió mucho, todo lo contrario, los problemas se agudizaron. Las contrariedades que estos niños y jóvenes sufrieron dentro, no sólo estuvieron relacionadas con la falta de alimento, vestimenta, mobiliario o personal, algunos expedientes nos hablan de los casos de abuso sexual. Por ejemplo, el delegado investigador Enrique Catalán, escribió sobre el caso de Joaquín:

${ }^{21}$ Madrigal, Los menores, pp. 46, 49.

${ }^{22}$ Madrigal, Los menores, pp. 46, 49. 
Desde pequeño estuvo en el orfanato de la ciudad de Puebla. Posteriormente lo ha perjudicado el medio de la Casa de Orientación para Varones que ha influido desfavorablemente sobre su conducta convirtiéndolo más y facilitándole sus prácticas homosexuales a las que seguramente ha tenido tendencias desde antes de su llega a dicho establecimiento. En la misma Casa de Orientación para Varones siguió cometiendo algunos hurtos y se fugó, llegando hasta tercero y aprender algo del oficio de zapatero [...] A su llegada a la Casa de Orientación, varios muchachos de los más grandes lo cogieron por la fuerza y abusaron de él; ahora todo el que quiere tener tratos homosexuales con este muchacho lo logra, la Casa de Orientación no es un lugar, ni de educación ni de orientación y más bien sirve para pervertir a los muchachos que por desgracia llegan ahi. $\mathrm{Y}$ se ha visto algunos de los niños que por cualquier circunstancia son enviados a aquel lugar, después de uno o dos años, salen hechos unos andrajos, sin pudor, recato ni vergüenza; no se respetan a sí mismos ni respetan a los demás, habituados completamente al robo con una alta escuela de éstos, puesto que los demás les enseñan y sin deseo de progresar, ser útiles a alguien siendo todo lo contrario, ansias de venganza, deseos de libertinaje y de prostituirse más y más... ${ }^{23}$

El caso de Joaquín no fue el único en donde se señalan los "vicios" y "perversiones" que estos niños y jóvenes adquirían en estos establecimientos. Por ejemplo, en el caso de Leopoldo se dijo:

...de suerte que los tres años que estuvo en la Casa de Orientación, fueron tres años inútiles y cuenta el menor que cuando llegó a dicho lugar no sabía ni conocía mucho

23 "Intento de robo", México, marzo de 1937, AGn, Consejo Tutelar de Menores Infractores (en adelante CTMI), caja 48, exp. 14122. Las cursivas son mías. 
de lo que ahora sabe, pues nunca había tenido relaciones homosexuales y allí las tuvo; nunca había fumado la marihuana, ni siquiera la conocía y ahí adquirió el vicio. ${ }^{24}$

Pero este tipo de instituciones no fueron las únicas en sufrir ese descuido por parte de las autoridades o del propio personal. Al leer las obras de Silvia Arrom, ${ }^{25}$ María Dolores Lorenzo ${ }^{26}$ o Emeterio Payá Valera, ${ }^{27}$ libros tan distintos entre sí, pero que describen la situación de diferentes instituciones que tuvieron como objetivo proteger a los más desvalidos. De tal forma, el Hospicio de Pobres, la Escuela EspañaMéxico y otras instituciones en diferentes épocas, tuvieron que enfrentar problemas parecidos -accidentes, fugas, sobrepoblación, robo, falta de ropa, de comida y de personal capacitado, etcétera-, que nos hacen pensar que la situación del Tribunal para Menores y de sus casas de corrección no tenían nada de extraordinario, todo lo contrario, es asombroso apreciar las similitudes que existen entre ellas.

Una constante que encontramos en los archivos, no sólo se refiere a la reincidencia, sino también a las fugas de los menores. De acuerdo con nuestra información de los 2162 expedientes, $18.50 \%$ de ellos informaba de la fuga de estos niños y jóvenes. Estos chicos, para lograr escapar, aprovechaban las labores que les eran asignadas tanto dentro como fuera de las correccionales. Por ejemplo, Eduardo se logró fugar cuando fue comisionado para ir por el pan a la penitenciaría; ${ }^{28}$ Jesús se escapó de la Casa de Observación cuando fue comisionado para tirar basura; ${ }^{29}$ Leopoldo logró huir con otro menor cuando estuvieron ayudando en la cocina, al subir por la azotea; ${ }^{30}$ por último, Carlos, que formaba parte de la banda de guerra, logró evadirse cuando fueron llevados a Mixcoac. ${ }^{31}$

\footnotetext{
24 "Delitos contra la salud", México, marzo de 1937, AGN, CTMI, caja 49, exp. 14358.

25 Arrom, Para contener, 2011.

${ }^{26}$ Lorenzo Río, El Estado, 2011.

${ }^{27}$ Payá, Los niños, 1985.

28 "Robo", México, marzo de 1939, AGn, ctmi, caja 49, exp. 14321.

29 "Robo", México, febrero de 1931, agn, cTMI, caja 21, exp. 6510.

30 "Homicidio", México, marzo de 1937, AGN, CTMI, caja 49, exp. 14358.

31 "Tráfico de marihuana”, México, mayo de 1936, AGN, CTMI, caja 42, exp. 12419.
} 
Otros vieron la oportunidad de huir en los eventos que la institución organizaba o en la que participaba. Es así que, Enrique logró escapar cuando se organizó un grupo de exploradores, ${ }^{32}$ Rito se fugó cuando participaron en un festival en honor al expresidente Plutarco Elías Calles; ${ }^{33}$ José y otros compañeros se evadieron después de haber terminado un festival "pro árbol" al mezclarse con el público. ${ }^{34}$ Estas fugas también llegaron a ser grupales. Por ejemplo, Arturo huyó con otros menores

...rompiendo un barrote de una de las puertas del salón de clases "Narciso Mendoza", y forzando a su vez la reja de una de las ventanas que dan a los Tribunales de Justicia, por donde lograron tomar calle. Esta maniobra fue efectuada a la hora en que el vigilante de turno, se encontraba en $[\mathrm{el}]$ comedor tomando su merienda. ${ }^{35}$

Otro elemento que permitía que los chicos y jóvenes lograran evadirse era debido a la falta de personal. En el caso de Rodolfo se comunicaba su fuga junto con Antonio, ambos estaban comisionados en la cocina, a cargo de la cocinera pero:

...como esta se distrajo, se evadieron por la verja que daba a las calles de Serapio Rendón. Estas fugas son muy frecuente[s] debido, en primer lugar, a que las bardas no han sido elevadas por falta de presupuesto en el Departamento Central y por otra parte a que hace un mes, no se ha presentado el empleador que la Secretaría de Gobierno había comisionado en este Centro para la vigilancia. ${ }^{36}$

\footnotetext{
32 "Robo", México, mayo de 1928, aGn, cTMI, caja 2, exp. 2105.

33 "Robo", México, agosto de 1929, AGN, cTMI, caja 4, exp. 2654.

34 "Robo", México, junio de 1929, AGN, CTMI, caja 22, exp. 6784.

35 "Robo", México, febrero de 1933, AGn, CTMI, caja 20, exp. 6281.

36 "Robo", México, julio de 1935, AGn, CTMI, caja 38, exp. 11035.
} 
El problema de estos centros no sólo radicaba en la falta del personal, sino también en las malas condiciones en que se encontraban los establecimientos. De la evasión de Alfonso con otros diez compañeros se señaló:

...en años anteriores este plantel contaba con un destacamento formado por 25 policías que se encargaban de la vigilancia y en la actualidad este servicio se cubre con tres policías que se turnan cada seis horas [...] así como también a que las bardas del edificio son bajas con algunos tramos de la casa en ruinas y que prácticamente son escaleras fáciles de aprovechar. ${ }^{37}$

La oportunidad de fugarse parece ser que era relativamente fácil, hasta podríamos preguntarnos si los mismos empleados no estaban confabulados con los menores para que ellos pudieron escapar. Algunos otros conseguían ganarse la confianza de las autoridades y obtenían permisos para salir y visitar a sus familiares, al principio regresaban los días establecidos, pero después simplemente desaparecían. Por ejemplo, a Otilio se le permitió ir de vacaciones pero no regreso; ${ }^{38}$ José consiguió el permiso para visitar a sus familiares, pero no volvió más, ${ }^{39}$ lo mismo pasó con Marcelino. ${ }^{40}$

Además de los problemas por los que atravesaba el Tribunal y sus instalaciones, encontramos ciertas anomalías administrativas, que tenían que ver con el trabajo de los menores y los permisos para salir. Cuando las y los jóvenes se acercaban a los dieciocho años o llevaban mucho tiempo en las correccionales, se les daba permiso para buscar trabajo. De tal forma, que cuando salieran de las instalaciones tuvieran un medio para valerse por sí mismos. Pero en nuestra revisión, encontramos que hubo permisos que se dieron para poder trabajar

\footnotetext{
37 "Robo", México, mayo de 1934, agn, ctMi, caja 30, exp. 8718.

38 "Robo", México, mayo de 1935, AGN, cTMI, caja 36, exp. 10603.

39 "Estupro", México, agosto de 1935, AGN, CTMI, caja 38, exp. 11084.

40 "Robo", México, septiembre de 1935, AGN, ctmi, caja 38, exp. 11263.
} 
fuera mientras permanecían en el establecimiento, es decir, este trabajo era complementario a su estancia en los establecimientos. Pero, además no tenían el tiempo ni la edad suficientes para buscar trabajo y lograr ser externados.

El otro problema, es el tipo de trabajo que se les permitió ejercer, para los reformadores sociales el trabajar en la calle era sinónimo de delincuencia, por tal motivo, ser canastero, bolero, vendedor de billetes, vendedor de periódicos, entre otros, eran trabajos mal vistos. En este caso, los expedientes de Delfino ${ }^{41}$ y Alfonso $^{42}$ son interesantes, porque ambos menores fueron enviados a la Casa Hogar que se encontraba en Revolución 110. Delfino por robo y Alfonso por ebrio, ambos menores fueron acusados de fuga, se decía que la Casa Hogar les había dado permiso para que con otros compañeros se dedicaran a la venta de periódicos. El por qué la Casa Hogar permitía este tipo de situaciones no queda claro, de lo que estamos seguros es que esta clase de permisos les daba la posibilidad a los chicos de salir y no regresar más.

La falta de presupuesto, de vigilancia y de personal capacitado para trabajar con los menores pudieron ser algunos de los factores que repercutieron en el buen funcionamiento de estas instituciones, como Carmen Madrigal denunció en su momento. Además, ella añadió, que los jueces del Tribunal habían hecho todo lo que estaba en sus manos para lograr la enmienda de los menores pero la falta de presupuesto les había impedido hacer más.

Asimismo, de todos los elementos ya mencionados también podemos señalar la sobrepoblación en estas instituciones. Algunos niños y jóvenes tuvieron que esperar en la Casa de Observación semanas o hasta meses antes de que fueran enviados a los establecimientos que les correspondían. Uno de los métodos utilizados por las autoridades para hacer espacio en estas instituciones, eran las famosas externaciones, nos referimos cuando las autoridades dejaban en libertad a los menores.

Para Elena Azaola, las externaciones sólo fueron ficciones creadas por la institución para poder legitimar su poder, es decir, eran ejemplos

\footnotetext{
41 "Robo", México, junio de 1935, AGN, ctmi, caja 37, exp. 10858.

42 "Ebrio", México, julio de 1935, AGN, CTMI, caja 38, exp. 11043.
} 
de la supuesta rehabilitación de los menores. De tal forma, si los menores regresaban a su hogar se debía a que ellos ya habían sido "regenerados" o "rehabilitados". Pero para esta autora, sólo eran una ficción creada desde arriba, es decir, planteada por las autoridades de las correccionales para justificar su lugar en la sociedad. De esta forma, las autoridades pudieron mantener un doble discurso. Por un lado, se mostró a la sociedad que las correccionales estaban cumpliendo con su función, es decir, la "rehabilitación" de los menores. Pero a su vez, admitían que estos niños podrían volver a reincidir. Lo más importante para ellos era "mantener una población relativamente constante" que pudieran controlar. ${ }^{43}$

Por otro lado, posiblemente esta falta de recursos y el descuido en que se encontraban los menores pudieron haber sido la causa de muerte de algunos de ellos. De los expedientes revisados encontramos que fueron cuatro los que murieron estando en el Tribunal, sin tomar en cuenta los bebés que llegaron a nacer en la Casa de Observación para mujeres. Leopoldo murió en 1934 debido a una congestión pulmonar, no se pudo encontrar a su familia; ${ }^{44}$ Ignacio murió al ser atropellado por el camión de la escuela, después de haber participado en un festival, iba en el estribo cuando se cayó, ${ }^{45}$ Pedro murió de una "intoxicación aguda", días antes se le había otorgado su externación pero había decidido permanecer en la Casa de Orientación para varones; ${ }^{46}$ en cambio, María de la Luz murió un mes después en el hospital al contraer tifo. ${ }^{47}$ Estos son los pocos registros que quedan, de hecho, Elena Azaola menciona de un menor que fue muerto por su profesor en la Escuela de Orientación para varones, desafortunadamente no pudimos consultar el expediente, pero estos casos nos hablan de la falta de personal capacitado para cuidar de los niños y las niñas que se encontraban en estos establecimientos.

\footnotetext{
${ }^{43}$ Azaola, La institución, p. 338.

44 "Robo", México, 1931, AGN, CTMI, caja 9, exp. 4039.

45 "Robo", México, junio de 1933. AGn, cTMI, caja 21, exp. 6649.

46 "Robo", México, junio de 1935, Agn, ctmi, caja 37, exp. 10821.

47 "Abandono de hogar", México, febrero de 1936, AGN, CTMI, caja 41, exp. 11920.
} 
Por ejemplo, aunque estaba prohibido hacer uso de la violencia y de castigar fuertemente a los menores, hay expedientes que describen este tipo de situación. Salvador, de 15 años de edad, había sido enviado a la Escuela Industrial por haber hurtado quince pañuelos. Dentro de la Escuela Industrial se dedicó a hurtar con otros compañeros, cuando las autoridades de la institución se dieron cuenta, interrogaron a los jóvenes. Uno de ellos confesó fácilmente - de acuerdo con el informe que fue enviado al Tribunal- pero para que Salvador lograra confesar "dos delegados del Tribunal [...] lo golpearon con dureza, lo mismo que el prefecto del establecimiento" después, fueron remitidos al Tribunal y "se le prohibió terminantemente hablar con los demás; más todavía, se les tuvo de plantón el primer día, se les 'avergonzó' delante de todos los muchachos regañándolos con palabras en extremo duras".

Casi al final del informe se señaló que el comportamiento de Salvador había mejorado "antes de una semana", posiblemente con esto se trataron de justificar las acciones de los delegados investigadores. ${ }^{48}$

Por otro lado, el papá de Octaviano también había pedido la externación de su hijo porque sabía que en la Casa de Orientación era maltratado, a pesar de esta denuncia la solicitud no procedió, y cuatro años después se comunicaba la fuga de Octaviano al "seguetear la reja de un tragaluz". ${ }^{49}$ En cambio, María Isabel se fugó de la Casa de Orientación para Mujeres y se presentó a la Jefatura de Policía en donde denunció que había sido maltratada, al parecer la denuncia no prosperó porque fue enviada a la Casa Hogar de donde se fugó tiempo después. ${ }^{50}$

\section{Reflexión final}

A principios del siglo $\mathrm{xx}$, el país tuvo que encarar las consecuencias de la Revolución mexicana, algunas de ellas causadas por el mismo conflicto - como la desestabilidad política y económica o la desintegración de las familias por la muerte de un familiar durante el conflicto-',

\footnotetext{
48 "Robo", México, 1928, Agn, ctmi, caja 13, exp. 3418.

49 "Robo", México, diciembre de 1933, AGN, CTMI, caja 28, exp. 8093.

50 "Por no tener familiares", México, junio de 1935, AGN, ctmi, caja 37, exp. 10836.
} 
o en todo caso en profundizar otros problemas causados por el crecimiento del país - la migración, la sobrepoblación, la modernización, la pobreza, etcétera-. De tal forma que las instituciones que aparecieron en estos años tuvieron como objetivo paliar o solucionar estos problemas. El Tribunal para Menores Infractores fue una de esas instituciones, su objetivo era solucionar o frenar la delincuencia infantil, pero como pudimos describir en este pequeño artículo, dicha institución estuvo lejos de alcanzar su objetivo.

Los problemas que enfrentó el Tribunal no fueron únicos, todo lo contrario, hay una gran similitud con otras instituciones, ya mencionadas, esta situación nos lleva a preguntar: ¿hubo o no fracaso del Tribunal? El análisis de los expedientes nos permite comprender el papel que la institución tuvo dentro de la sociedad durante esta época, debido a que algunos padres enviaban a sus hijos con la esperanza de que aprendieran un oficio y se forjaran un camino que les permitiera vivir honradamente y ser "útiles a la sociedad". Estos casos nos permiten ver los diferentes usos que se hicieron de esta institución, debido a que también se hizo cargo de niños o jóvenes abandonados o huérfanos, y de los llamados incorregibles, es decir, menores que no atendían a la autoridad paterna, que se caracterizaban por su rebeldía, su irresponsabilidad y falta de interés por la escuela. Estos padres esperaban que dentro de esta institución pudieran corregir su comportamiento.

De tal forma, el Tribunal para Menores, en términos generales, estuvo lejos de cumplir con su objetivo general. Pero si se analizan los casos particulares, principalmente de aquéllos que se acercaron al Tribunal buscando ayuda, esta institución cumplió con sus funciones y con sus acciones, justificó su papel dentro de la sociedad, fue una institución que palió momentáneamente, o algunas veces hasta permanente, el abandono social de muchos niños y jóvenes de la Ciudad de México.

La complicación fundamental del Tribunal fue, como ya lo mencionamos, que sólo resolvía los problemas momentáneamente, no los atacaba de raíz. El Tribunal no logró que los menores se corrigieran al cien por ciento, pero sí formó parte de las estrategias de sobrevivencia de muchas familias y niños y jóvenes de la Ciudad de México. 
Fue un espacio donde pudieron interactuar y negociar niños, familiares y autoridades. De esta manera, si analizamos al Tribunal desde esta óptica, podemos decir que cumplió con sus funciones secundarias, cobijó, alimentó y enseñó a aquellos niños que estaban abandonados socialmente. Asimismo, estos casos nos muestran que los niños y jóvenes de las clases pobres de la Ciudad de México no fueron sujetos pasivos, moldeables y fáciles de dirigir, como llegaron a pensar los jueces e intelectuales de la época. Todo lo contrario, fueron niños y jóvenes que supieron hacer frente a la adversidad, se comportaban de acuerdo con las exigencias de los adultos, fingían acatar las reglas pero, en el menor descuido desaparecían.

Son pocos los estudios que analizan la participación de estos niños y jóvenes en la sociedad pero, el análisis de los expedientes del Tribunal para Menores nos abre una serie de ventanas para aproximarnos a ellos. En lo que cabe, este texto buscó la forma de acercarse, tanto a los problemas de la institución como a la participación de los menores ante su situación. De tal modo que si tenemos que responder a nuestra pregunta, ¿hubo o no fracaso del Tribunal?, la respuesta sería no. Esta institución nació con un fin, pero en el camino tuvo que ir resolviendo, con la práctica y con la experiencia de los jueces, problemas que posiblemente no se tenían contemplados. Esta institución se convirtió en una opción para resguardar a la infancia huérfana y abandonada socialmente. 


\section{Fuentes}

\section{Archivos}

Archivo General de la Nación (AGN).

\section{Hemerografía}

Diario Oficial, Ciudad de México.

El Universal, Ciudad de México.

\section{Bibliografía}

Alcubierre Moya, Beatriz, "La infancia en las Conferencias Panamericanas en los años veinte: una historia común", en Carlos Marichal (coord.), México y las Conferencias Panamericanas, 1889-1938. Antecedentes de la globalización, México, Secretaría de Relaciones Exteriores, 2001.

Arrom, Silvia Marina, Para contener al pueblo: el Hospicio de Pobres de la Ciudad de México (1774-1870), México, Publicaciones de la Casa Chata/CiEsas, 2011.

Azaola, Elena, La institución correccional en México. Una mirada extraviada, México, Siglo XXI, 1990.

Beteta, Ramón, Jarano, México, Fondo de Cultura Económica, 1989. , La mendicidad en México, México, Beneficencia Pública en el Distrito Federal, Departamento de Acción Social, 1931.
A. Mijares y Hno., 1935. 
Lorenzo Río, María Dolores, El Estado como benefactor. Los pobres y la asistencia pública en la Ciudad de México, 1877-1905, México, El Colegio de México/El Colegio Mexiquense, 2011.

Madrigal, Carmen, Los menores delincuentes. Estudio sobre la situación de los tribunales para menores. Doctrina y realidad, México, Ediciones Botas, 1938.

Netto Nunes, Eduardo Silveira, "La infancia latinoamericana y el Instituto Internacional Americano de Protección a la Infancia (1916-1940)", en Susana Sosenski y Elena Jackson (coords.), Nuevas miradas a la historia de la infancia en América Latina. Entre prácticas y representaciones, México, Universidad Nacional Autónoma de México, 2012, pp. 273-302.

Payá, Emeterio, Los niños españoles en Morelia: el exilio infantil en México, México, Edamex, 1985.

Pérez García, Juan Martín, "La infancia callejera: paradigma de la discriminación tutelar", en Manfred Liebel y Marta Martínez Muñoz (coords.), Infancia y derechos humanos. Hacia una ciudadania participante y protagónica, Perú, Instituto de Formación para Educadores de Jóvenes, Adolescentes y Niños Trabajadores de América Latina y el Caribe, 2009, pp. 295-342.

Piccato, Pablo, Ciudad de sospechosos: crimen en la Ciudad de México, 1900-1931, México, Publicaciones de la Casa Chata/ciesas/Conaculta, 2010.

Shell, Patience A., "Nationalizing Children through Schools and Hygiene: Porfirian and Revolutionary Mexico City", The Americas, vol. 60, núm. 4, abril de 2004, pp. 559-587.

Sosenski, Susana, "Infancia y familias posrevolucionarias", en Legajos Boletín del Archivo General de la Nación, núm. 1, julio-septiembre de 2009, pp. 10-26. 
Trujillo Bretón, Alberto, 'Los hijos del arroyo en la 'Ciudad del Polvo', Guadalajara, 1876-1933”, en Antonio Padilla (coords.), La infancia en los siglos XIX y Xx. Discursos e imágenes, espacios y prácticas, México, Casa Juan Pablos/Universidad Autónoma del Estado de Morelos, Ediciones Mínimas, 2008, pp. 280-406.

Urías Horcasitas, Beatriz, "Degeneración e higiene mental en el México posrevolucionario, (1920-1940)", en Frenia. Revista de Historia de la Psiquiatría, vol. 2, núm. 4, 2004, p. 38. 